\title{
The Effect of Intravenous Tranexamic Acid on Hemoglobin and Hematocrit Levels After Cesarean Delivery: a Randomized Controlled Clinical Trial
}

Esmat Jafarbegloo ( $\sim$ jafarbegloo_2004@yahoo.com )

Qom University of Medical Sciences

Faride Faridnyia

Qom University of Medical Sciences

Atefeh sadat mohammad hoseini nejad

Qom University of Medical Sciences

\section{Research}

Keywords: hemoglobin, hematocrit, Tranexamic acid, Cesarean section, Randomized controlled trials

Posted Date: August 30th, 2021

DOl: https://doi.org/10.21203/rs.3.rs-838710/v1

License: (1) (i) This work is licensed under a Creative Commons Attribution 4.0 International License.

Read Full License 


\section{Abstract}

Background: During a cesarean section, the mother loses a lot of blood. This bleeding can cause a drop in hemoglobin and hematocrit. Tranexamic acid, an anti-fibrinolytic agent, is a novel approach in an attempt to prevent this dreadful complication. This study aims to explore the effect of intravenous tranexamic acid on hemoglobin and hematocrit level after cesarean section(CS) in women who were low risk in postpartum hemorrhage.

Methods: This prospective randomised placebo-controlled clinical trial was carried out on 50 pregnant women referred to Izadi hospital, Qom, Iran, 2017. women were divided into two groups of 25 cases using block randomization. The study group, 25 women, received tranexamic acid 10 minutes before CS whereas the control group, 25 women received distilled water. Hemoglobin and hematocrit levels were measured before and 12-24 hours after delivery.

Results: There was no significant difference in pre-operative and 12-24 h post-operative hemoglobin and hematocrit levels between the two groups $(p>0.05)$.

Conclusions: Pre-operative prescription of $1 \mathrm{gr}$ tranexamic acid was not associated with improvement of post-operative hemoglobin and hematocrit.

Trial registration: IRCT20091010002558N7.

\section{Plain English Summary}

Blood loss during cesarean section can led to drop in hemoglobin and hematocrit levels. Tranexamic acid inhibits fibrinolysis at the site of bleeding and has been used to decrease bleeding during cesarean delivery. The aim of the present study was to assess the effect of intravenous tranexamic acid on hemoglobin and hematocrit levels after cesarean delivery.

In our study the mean of $\mathrm{Hb}$ drop before and after intervention in the study group was lower than the control grous .Although the difference between the two groups was not statistically significant, it does seem to be clinically important.

\section{Background}

\section{Prevention of Postpartum haemorrhage}

Postpartum haemorrhage (PPH) is the third-most common cause of maternal death in the United States and it is still the first prevalent cause of maternal death in developing countries(1). PPH is classically defined as the loss of more than $500 \mathrm{ml}$ of blood following vaginal delivery or more than $1000 \mathrm{ml}$ loss following caesarean section(CS)(2). Cesarean section rate has increased to as high as 25 to $30 \%$ in many countries of the world. Hemorrhage and anemia can be a serious complication of cesarean delivery. In 
order to decrease maternal morbidity and mortality caused by bleeding and hemoglobin drop, reduction of this severe bleeding and anemia is vital $(3,4)$.

\section{Tranexamic Acid}

Antifibrinolytic agents, mainly tranexamic acid (TA), have been demonstrated to reduce blood loss and transfusion requirements in various surgical procedures, such as coronary artery bypass, scoliosis surgery and knee arthroplasty.(4-8) In the field of obstetrics, many randomised, controlled trials and meta-analysis have suggested that TA administration in women after vaginal or elective caesarean delivery reduces blood loss and the incidence of $\mathrm{PPH}[3,9-19]$. But there are few studies on the effect of tranexamic acid on Hemoglobin and Hematocrit Levels after Delivery. Therefore, in this study, the effect of tranexamic acid on hemoglobin and hematocrit levels after cesarean delivery was investigated.

\section{Material And Methods}

A randomized case-controlled clinical trial was conducted on 50 women undergoing cesarean, at Izadi Hospital, Qom, Iran, between August 15, 2016, and April 30, 2017. The study was approved by the ethics committee of Qom University of Medical Sciences (code: MUQ.REC.1394.154). It was prospectively registered with the Iranian registry of clinical trials (code: IRCT20091010002558N7). Written informed consent was obtained from all participants.

\section{Inclusion criteria:}

Pregnant women who were to undergo elective cesarean delivery by Pfannenstiel incision under spinal anesthesia were eligible for inclusion in the study. Inclusion criteria were women aged 18-35 years with a singleton pregnancy at 38 weeks and 42 weeks' gestation and Blood pressure less than 140/90 mmHg.

\section{Exclusion criteria:}

Pregnancy complications such as pre-eclampsia, polyhydramnios, macrosomia, preterm labour, multiple pregnancies, placenta praevia, abruptio placenta, abnormal placenta, thrombophilia, anemia, coagulopathy, cardiovascular, renal, liver disorders and allergy to tranexamic acid were excluded from the study.

\section{Sample size:}

Sample size was calculated that 22 subjects would be required per group in order to detect this difference with $80 \%$ power and $5 \%$ probability of type 1 error. Adjusting for drop out, recruitment target was set at 25 subjects per group. Figure 1 shows summary of study design.

\section{Random allocation\& Intervention:}

The women were randomly allocated into two groups based on the block randomization: Women in the study group $(n=25)$, received one gram intravenous TA, 10 min before skin incision, and the control 
group $(n=25)$, received one gram placebo (distilled water) in $200 \mathrm{~mL}$ normal saline over $10 \mathrm{~min}$. In this study, TA was supplied in $2 \times 500 \mathrm{mg} / 5 \mathrm{~mL}$ ampoules obtained from kharazmi Pharmaceutical Company in Iran. The placebo comprised $2 \times 5 \mathrm{ml}$ distilled water ampoules from Shahid Ghazi Pharmaceutical Company in Tabriz - Iran.

In order to hide the medication allocation, two vials of the tranexamic acid and distilled water were placed in similar opaque sequentially numbered sealed packages by an Operator room technician that not involved in sampling and analysis, who maintained the medication administration code. In this way, the data evaluators and participants had no knowledge of the study medication.

\section{Outcome measures:}

Haemoglobin and haematocrit levels were measured before and 12-24 h after cesarean delivery.

Both groups received $20 \mathrm{IU}$ oxytocin in $1000 \mathrm{~mL}$ of normal saline over 20 min after placental delivery. The dose of oxytocin received and other additional uterotonic administered were recorded for both groups.

Vital signs (heart rate, blood pressure and respiratory rate) were recorded before operation, 1 and $2 \mathrm{~h}$ after cesarean delivery. Complete blood count (CBC) was performed $12-24 \mathrm{~h}$ after delivery. Creatinine ,BUN and urine analysis were performed before delivery, and 48-72 hours after delivery.

\section{Statistical analysis:}

The data were analysed using SPSS/version 17 (IBM SPSS, New York, NY, USA) and t-test was used to compare differences between the two groups were described with $95 \%$ confidence intervals $(95 \% \mathrm{Cl})$, and $p$-value 0.05 was considered significant statistically.

\section{Results}

The women characteristics in the two groups were similar, with no statistical difference between the two groups (Table 1). 
Table 1

Women characteristics for study and control group

\begin{tabular}{|llll|}
\hline characteristics & $\begin{array}{l}\text { Study } \\
\text { mean } \pm \text { SD }\end{array}$ & $\begin{array}{l}\text { Control } \\
\text { mean } \pm \text { SD }\end{array}$ & p-value \\
\hline age & $30.48 \pm 4.71$ & $31.46 \pm 4.85$ & 0.973 \\
\hline Hight & $159.76 \pm 5042$ & $160.18 \pm 7.33$ & 0.894 \\
\hline Weight & $64.92 \pm 14.45$ & $67.71 \pm 14.18$ & 0.906 \\
\hline BMl & $25.31 \pm 4.94$ & $26.43 \pm 4.75$ & 0.930 \\
\hline Weight gain & $10.52 \pm 4.03$ & $12.72 \pm 4.07$ & 0.852 \\
\hline Gravida & $2.32 \pm 0.74$ & $2.29 \pm 0.80$ & 0.739 \\
\hline Pariety & $1.16 \pm 0.55$ & $1.13 \pm 0.61$ & 0.818 \\
\hline Gestational age & $38.24 \pm 0.436$ & $37.83 \pm 1.76$ & 0.274 \\
\hline Number of CS & $1.21 \pm 0.50$ & $1.04 \pm 0.62$ & 0.563 \\
\hline
\end{tabular}

No significant difference regarding pre-operative hemoglobin and hematocrit value was found, in both groups. The hemoglobin and hematocrit values decreased slightly after CS in the two groups, but there was no statistical difference. The mean of hemoglobin and hematocrit drop before and after intervention was not significant difference between both groups(Table2).

Table 2

The measured main outcomes in both studied groups.

\begin{tabular}{|llll|}
\hline Variables & $\begin{array}{c}\text { Study group } \\
(\text { mean } \pm \text { SD) }\end{array}$ & $\begin{array}{l}\text { Control group } \\
(\text { mean } \pm \text { SD) }\end{array}$ & P value \\
\hline Pre-operative $\mathrm{Hb}^{*}$ level $(\mathrm{mg} / \mathrm{dl})$ & $12.17 \pm 1.21$ & $13.35 \pm 1.37$ & 0.456 \\
\hline 12-24 h post-operative $\mathrm{Hb}$ level $(\mathrm{mg} / \mathrm{dl})$ & $11.72 \pm 0.99$ & $11.41 \pm 1.6$ & 0.43 \\
\hline Mean of Hb drop before and after intervention & $0.458 \pm 0.75$ & $0.945 \pm 1.25$ & 0.11 \\
\hline Pre-operative Hct ${ }^{* \star}$ value $(\%)$ & $37.73 \pm 2.38$ & $37.70 \pm 3.45$ & 0.17 \\
\hline 12-24h post-operative Hct value(\%) & $36.22 \pm 2.41$ & $37.31 \pm 3.04$ & 0.15 \\
\hline Mean of Hct drop before and after intervention & $1.508 \pm 2.41$ & $0.395 \pm 3.34$ & 0.42 \\
\hline *Hb: hemoglobin **Hct: hematocrit & & & \\
\hline
\end{tabular}


There was no significant difference in the BUN, $\mathrm{Cr}$ and urine analysis before and48-72h after CS between the two groups.

There was no significant difference in the vital signs (heart rates, respiratory rates and blood pressures) before and 1-2 hours after CS between the two groups.

There was no significant difference in the APGAR scores at $1 \mathrm{~min}$ and also at $5 \mathrm{~min}$ between the two groups.

No side effects of tranexamic acid such as nausea, vomiting and diarrhea were reported in the study group.

There were no episodes of thrombosis in any of the women in the study group.

No PPH was reported and also, no blood transfusion was needed in each group in our study.

Additionally, There was no statistical difference in the additional uterotonic drugs between the two groups.

\section{Discussion}

The present study demonstrated that consumption of $1 \mathrm{gr}$ intravenous tranexamic acid $10 \mathrm{~min}$ before skin incision, did not make a significant difference in 12-24 h post-operative hemoglobin and hematocrit value between the two groups. Despite the Mean of $\mathrm{Hb}$ drop before and after intervention between the two groups were not significantly different, However, the mean of $\mathrm{Hb}$ drop before and after intervention in the study group was lower than the control group $(0.458 \pm 0.75$ vs $0.945 \pm 1.25)$ and this finding is clinically important.

There are few studies on the effect of tranexamic acid on Hemoglobin and Hematocrit Levels after Delivery.

A similar study was carried out by Mirghafoorvand et al. [20] To evaluate the The Effect of Intravenous Tranexamic Acid on Hemoglobin and Hematocrit Levels after Vaginal Delivery. After intervention, mean of hemoglobin loss was $1.4 \pm 0.9 \mathrm{~g} / \mathrm{dl}$ in intervention group and $1.7 \pm 1.0 \mathrm{~g} / \mathrm{dl}$ in control group $(p=0.11)$. The mean of hemoglobin before and after intervention was not statistical difference between two groups, but The mean of hematocrit loss was $3.7 \pm 2.3$ in intervention group and $4.8 \pm 3.0$ in control group $(p=0.03)$.

According to the study was carried out by Loic Sentilhes et al. [21] $1 \mathrm{gr}$ of tranexamic acid or placebo, administered intravenously, in addition to prophylactic oxytocin after delivery. they showed that among women with vaginal delivery who received prophylactic oxytocin, the use of tranexamic acid did not result in a rate of postpartum hemorrhage of at least $500 \mathrm{ml}$ that was significantly lower than the rate with placebo. Also there were no statistical difference in peripartum change in haemoglobin and haematocrit levels between the two groups. These results were consistent with our study. 
Another study was carried out by Movafegh et al.[3] by administering $10 \mathrm{mg} / \mathrm{kg}$ tranexamic acid 20 minutes before skin incision at cesarean delivery. Blood loss was significantly lower in the TA group than in the control group. Also, there was no significant difference in pre-operative and 24 hours post-operative haemoglobin levels between the study group and control group. These results were similar to our study.

Irene ray et al. [16] performed their study with intravenous administration of $1 \mathrm{gr}$ IV tranexamic acid 20 min before skin incision at caesarean delivery and found that the mean intra-operative and post-partum blood loss were significantly lower in the study group than the control group. The difference between the pre-operative and post-operative hemoglobin levels was significantly less in the study group than the control group. The decrease in hemoglobin in the study group could be due to the fact that, the mean of pre-operation hemoglobin was $10.33 \pm 1.26$ in study group and $9.80 \pm 1.34$ in control group $(p=0.05)$. This may be due to the fact that the hemoglobin level in the control group was lower from the beginning.

Another study was carried out by Amr yehia et al. [22] To evaluate the efficacy of tranexamic acid in reduction of blood loss during and after cesarean section. Twenty four hours post-operative hemoglobin level was significantly higher in study group compared to control, also 24 hours post-operative hematocrit was significantly higher in study group compared to control. These results may be due to the fact that they injected tranexamic acid after delivery of the baby. We hypothesized that this may be due to the time of tranexamic acid administration .

There were no abnormalities in $\mathrm{CBC}$, urinalysis, renal function tests before or after tranexamic acid administration. These results were comparable with the study by Movafegh et al. [3],Irene ray et al.[16] and Gai et al. [4].

Also, in our study no significant abnormal vital signs occurred after TA administration as HR, BP, RR. This has been corroborated by other studies Movafegh et al. [3], Gai et al. [4], Jianjun Xu et al.[12] and Irene ray[16].

Side effects of intravenous tranexamic acid such as nausea, vomiting and diarrhoea was similar in both groups. These results were similar to previous studie [16].

In our study no PPH was reported. patients who had blood loss more than $1000 \mathrm{ml}$ were defined as PPH in our study. While, PPH has been defined as more than $400 \mathrm{ml}$ blood loss in the studies of Gai et al. [4] and Yang et al.[23] and more than $500 \mathrm{ml}$, in the study of Jianjun Xu et al.[12].

Additionally, oxytocin and other uterotonic drugs administration were not statistically difference in both groups. While, in the study of Movafegh et al Oxytocin administration was significantly less in the study group compared with the control group[3].

The incidence of thrombosis during pregnancy and puerperium is 5-6 times higher than that in the general population. When the antifibrinolytic drug tranexamic acid is administered, the increased risk of thrombosis should be considered, especially in the CS post-partum population $[4,16]$. In our study, 
however, none of the mothers reported signs of thrombosis. Similar results were found in other studies $[3-5,12,16]$.

All data demonstrated that TA can be used safely without increasing the occurrence of thrombosis, but still more researches are needed in this regard.

The safety of giving TA $(1 \mathrm{~g})$ while the foetus was still in utero was an important concern. As a consequence, the neonatal outcome was accurately evaluated by a neonatologist. In our study, there was no significant difference in the APGAR scores at $1 \mathrm{~min}$ and also at $5 \mathrm{~min}$ between the two groups. None of the neonates required NICU admission. Results were comparable to previous studies $[4,16]$.

There exist limitations to the present study. First, only English or persian articles were obtained. second, in our study the cosumption the TA was found to be safe and did not increase the risk for thrombogenic episodes in the mother. However, the study was not powered to test these aspects of the drug. A large, international study should be implemented to investigate the safety of TA in future research.

\section{Strengths:}

- This study provides detailed information on hemoglobin and hematocrit levels in relation to tranexamic acid intake.

\section{Limitations:}

- Only English or persian articles were obtained.

- in our study the cosumption the TA was found to be safe and did not increase the risk for thrombogenic episodes in the mother. However, the study was not powered to test these aspects of the drug.

\section{Conclusions}

Post-operative hemoglobin and hematocrit levels were not significantly different between the two groups and Pre-operative prescription of $1 \mathrm{gr}$ tranexamic acid was not associated with improvement of postoperative hemoglobin and hematocrit. Many studies have shown that TA consumption can reduce blood loss after delivery (9-19). In our study the mean of $\mathrm{Hb}$ drop before and after intervention in the study group was lower than the control group $(0.458 \pm 0.75$ vs $0.945 \pm 1.25)$. Although the difference between the two groups was not statistically significant, it does seem to be clinically important.

\section{Abbreviations}

CS: Cesarean section; PPH: Postpartum haemorrhage; TA: Tranexamic acid; CBC: Complete blood count; BUN: Blood urea nitrogen; $\mathrm{Cl}$ : confidence interval; SD: standard deviation; $\mathrm{Hb}$ : hemoglobin; $\mathrm{Hct:}$ hematocrit; Cr: Creatinine; APGAR: Appearance, Pulse, Grimace, Activity, and Respiration; NICU: neonatal intensive care unit. 


\section{Declarations}

Ethics approval and consent to participate: The study was approved by the ethics committee of Qom University of Medical Sciences (code: MUQ.REC.1394.154). It was prospectively registered with the Iranian registry of clinical trials (code: IRCT20091010002558N7). Written informed consent was obtained from all participants. Helsinki metrics were respected.

Consent for publication: Not applicable.

Availability of data and materials: The datasets generated and/or analysed during the current research are not publicly available as individual privacy could be compromised but are available from the corresponding author on reasonable request.

Competing interests: The authors declare that they have no competing interests.

Funding: This Study has been funded by Qom University of Medical Sciences (Code Number: MUQ.REC.1394.154).

Authors' contributions: Study concept and design: Esmat Jafarbegloo . Sampling: Faridnyia and Mohammad hoseini nejad. Analysis and interpretation of data: Faridnyia. Drafting of the manuscript: jafarbegloo. Critical revision of the manuscript for important intellectual content: Esmat Jafarbegloo. All authors read and approved the final manuscript.

Acknowledgements: This study is part of a research project that has been registered in the Clinical Trial Center with code IRCT 201602232558N6R1. We thank Mrs. Arezoo Bahadori for substantial help with this study.

\section{References}

1. Sahhaf F, Abbasalizadeh S, Ghojazadeh M, Velayati A, Khandanloo R, Saleh P, Piri R, Naghavi-Behzad M. Comparison effect of intravenous tranexamic acid and misoprostol for postpartum haemorrhage. Niger Med J. 2014; 55(4): 348-353.

2. WHO guidelines for the management of postpartum haemorrhage and retained placenta. Available from: http://whqlibdoc.who.int/publications/2009/9789241598514_eng.pdf

3. Movafegh A, Eslamian L, Dorabadi A. Effect of intravenous tranexamic acid administration on blood loss during and after cesarean delivery. International Journal of Gynecology and Obstetrics. 2011;115: 224-226.

4. Gai MY, Wu LF, Su QF et al. Clinical observation of blood loss reduced by tranexamic acid during and after caesarian section: a multi-center, randomized trial. Eur J Obstet Gynecol Reprod Biol. 2004; 112 (2): 154-157.

5. Sekhavat L, Tabatabaii A, Dalili M, Farajkhoda T, Dehghani Tafti A. Efficacy of tranexamic acid in reducing blood loss after cesarean section. The Journal of Maternal-Fetal and Neonatal Medicine. 
2009; 22(1): 72-75.

6. Ducloy-Bouthors AS, Jude B, Duhamel A, Broisin F, Huissoud C, Keita-Meyer H, Mandelbrot L, Tillouche N, Fontaine S, Goueff F, Depret-Mosser S, Vallet B. High-dose tranexamic acid reduces blood loss in postpartum haemorrhage. Critical Care. 2011; 15(117):1-10.

7. Xia Y, Griffiths BB, Xue Q. Tranexamic acid for postpartum hemorrhage prevention in vaginal delivery: A meta-analysis. Medicine. 2020;99(3):1-7.

8. Mielke R , Obermeyer S. The Use of Tranexamic Acid to Prevent Postpartum Hemorrhage. Journal of Midwifery \&Women's Health. 2020;65(3):410-416.

9. Gungorduk K, Asıcıoğlu O, Yıldırım G,Ark C, Tekirdağ A, Besımoglu B. Can Intravenous Injection of Tranexamic Acid Be Used in Routine Practice with Active Management of the Third Stage of Labor in Vaginal Delivery? A Randomized Controlled Study. Am J Perinatol 2013;30:407-414.

10. Sentilhes L ,Daniel V, Darsonval A, Deruelle P, Vardon D, PerrotinF, Ray C, Senat M, Winer N, Maillard F, Deneux-Tharaux C. Study protocol. TRAAP - TRAnexamic Acid for Preventing postpartum hemorrhage after vaginal delivery: a multicenter randomized,double-blind, placebo-controlled trial. BMC Pregnancy and Childbirth. 2015; 15(135): 1-13.

11. Alam A, Choi S. Prophylactic use of tranexamic acid for postpartum bleeding outcomes: a systematic review and meta-analysis of randomized controlled trials. Transfus Med Rev. 2015;29:231-41.

12. Xu J, Gao W, Ju Y. Tranexamic acid for the prevention of postpartum hemorrhage after cesarean section: a double-blind randomization trial. Arch Gynecol Obstet .2013; 287:463-468.

13. Ker K, Shakur H, Roberts I. Does tranexamic acid prevent postpartum haemorrhage? A systematic review of randomised controlled trials. BJOG. 2016;123:1745-1752.

14. Simonazzi G, Bisulli M, Saccone G, Moro E, Marshall A, Berghella V. Tranexamic acid for preventing postpartum blood loss after cesarean delivery: a systematic review and meta-analysis of randomized controlled trials. Acta ObstetGynecol Scand. 2016;95:28-37.

15. Roy P, Sujatha M. S, Bhandiwad A, Biswas B. Role of Tranexamic Acid in Reducing Blood Loss in Vaginal Delivery. The Journal of Obstetrics and Gynecology of India. 2016;66:246-250.

16. Ray I, Bhattacharya R, Chakraborty S, Bagchi C, Mukhopadhyay S. Role of Intravenous Tranexamic Acid on Caesarean Blood Loss: A Prospective Randomised Study. The Journal of Obstetrics and Gynecology of India. 2016; 66:347-352.

17. Heesen M, Böhmer J, Klöhr S, Rossaint R, Velde M, Dudenhausen J. W, Straube S. Prophylactic tranexamic acid in parturients at low risk for post-partum haemorrhage: systematic review and metaanalysis. Acta Anaesthesiol Scand. 2014; 58: 1075-1085.

18. Bouthors A, Hennart B, Jeanpierre E, Baptiste A, Saidi I, Simon E, Lannoy D, Duhamel A, Allorge D and Susen $S$. Therapeutic and pharmaco-biological, dose ranging multicentre trial to determine the optimal dose of Tranexamic acid to reduce blood loss in haemorrhagic cesarean delivery (TRACES): study protocol for a randomised, double-blind, placebo controlled trial. Trials; 2018; 19(148):1-13. 
19. Alam, A; Bopardikar, A; Au, S; Barrett, J; Callum, J; Kiss, A; Choi, S. Protocol for a pilot, randomised, double-blinded, placebo-controlled trial of prophylactic use of tranexamic acid for preventing postpartum haemorrhage (TAPPH-1).BMJ Open. 2017;7: 1-7.

20. mirghafoorvand M, Alizadeh Charandabi M, Abasalizadeh S, Shirdel F. The Effect of Intravenous Tranexamic Acid on Hemoglobin and Hematocrit Levels after Vaginal Delivery: A Randomized Controlled Trial. The Iranian Journal of Obstetrics, Gynecology and Infertility.2013;16 (60):1-8.

21. Sentilhes L, Azria E, Senat M, Ray C, Vardon D, Perrotin F. Tranexamic Acid for the Prevention of Blood Loss after Vaginal Delivery. N Engl J Med. 2018;379:731-42.

22. Yehia A, Koleib M, Abdelazim I, Atik A. Tranexamic acid reduces blood loss during and after cesarean section: A double blinded, randomized, controlled trial. Asian Pacific Journal of Reproduction. 2014; 3(1): 53-56.

23. Yang $\mathrm{H}$, Zheng S, Shi C. Clinical study on the efficacy of tranexamic acid in reducing postpartum blood loss: a randomized, comparative, multicenter trial. Zhonghua Fu Chan Ke Za Zhi $.2001 ; 36(10): 590-592$.

\section{Figures}




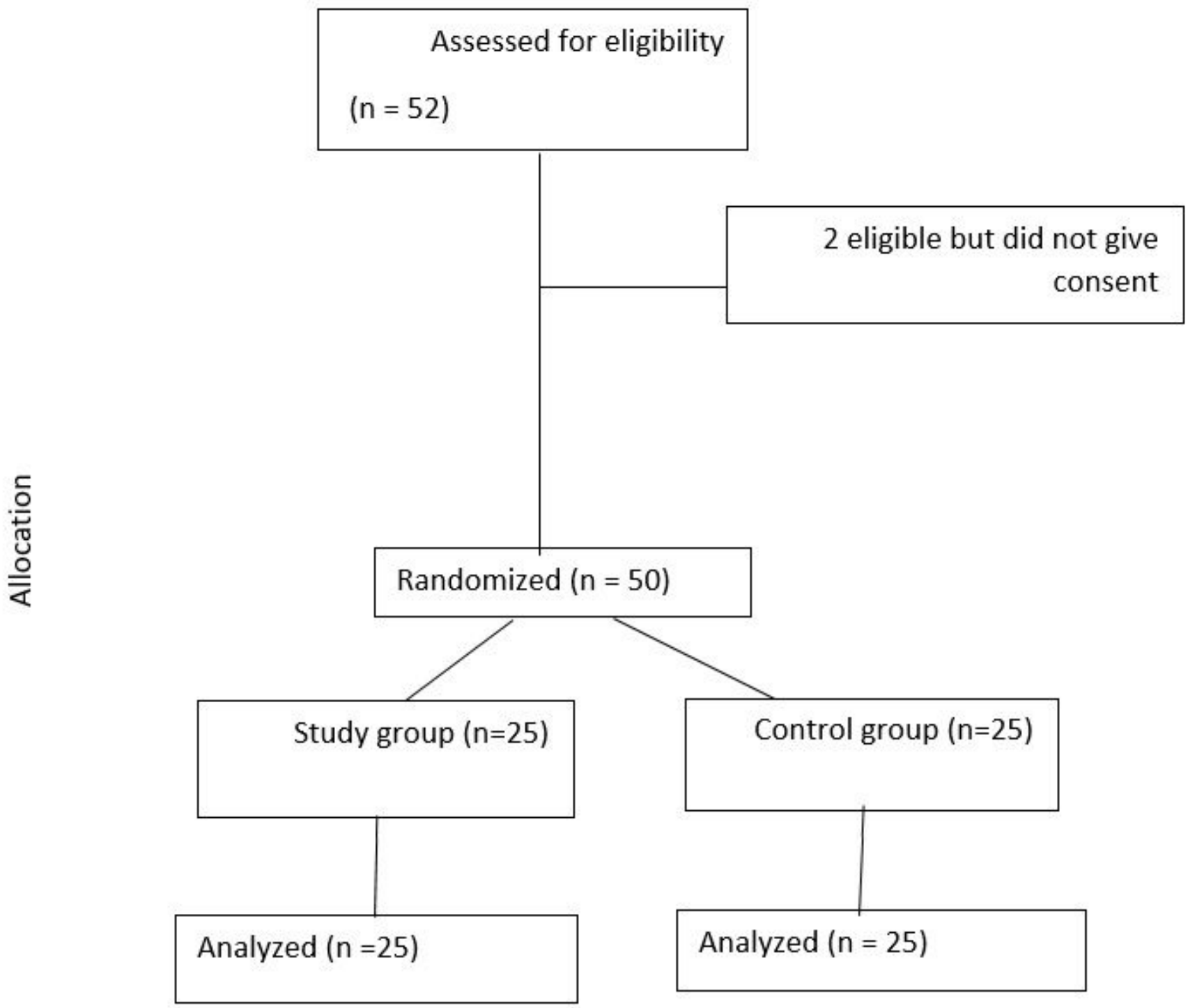

Figure 1

Flowchart of the study selection 